\title{
Estimating the Collapse Pressure of an Inflatable Aerodynamic Decelerator*
}

\author{
Frank E. Baginski ${ }^{\dagger}$ \\ The George Washington University, Washington, DC, 20052, USA \\ Kenneth A. Brakke \\ Susquehanna University, Selinsgrove, PA, 17870, USA, \\ Juan R. Cruz ${ }^{\S}$ \\ NASA Langley Research Center, Hampton, VA 23681, USA
}

\begin{abstract}
The collapse pressure of an inflatable membrane is the minimum differential pressure which will sustain a specific desired shape under an applied load. In this paper, we present a method for estimating the collapse pressure of a tension-cone inflatable aerodynamic decelerator (IAD) that is subject to a static aerodynamic load. The IAD surface is modeled as an elastic membrane. For a given aerodynamic load and sufficiently high torus differential pressure, the IAD assumes a stable axisymmetric equilibrium shape. When the torus pressure is reduced sufficiently, the symmetric equilibrium state becomes unstable and we define this instance to be the critical pressure $p_{c r}$. In this paper, we will compare our predicted critical torus pressure with the corresponding observed torus collapse pressure (OTCP) for fifteen tests that were conducted by the third author and his collaborators at the NASA Glenn Research Center $10^{\prime} \times 10^{\prime}$ Supersonic Wind Tunnel in April 2008. One of the difficulties with these types of comparisons is establishing the instance of torus collapse and determining the OTCP from quantities measured during the experiment. In many cases, torus collapse is gradual and the OTCP is not welldefined. However, in eight of the fifteen wind tunnel tests where the OTCP is well-defined, we find that the average of the relative differences $\left(\left|p_{c r}-\mathbf{O T C P}\right| / p_{c r}\right)$ was $\mathbf{8 . 9 \%}$. For completeness, we will also discuss the seven tests where the observed torus collapse pressure is not well-defined.
\end{abstract}

\section{Nomenclature}

$\mathcal{S} \quad$ Complete membrane.

$\mathcal{S}_{F} \quad$ Fundamental section of membrane $\mathcal{S}$.

$C_{A} \quad$ Axial force coefficient.

E Young's modulus of membrane (GPa).

$\nu \quad$ Poisson's ratio of membrane.

$h \quad$ Membrane thickness (micron).

* Submitted for presentation at the 22nd AIAA Aerodynamic Decelerator Systems Technology Conference and Seminar, 25-28 March 2013, Daytona Beach, Florida.

${ }^{\dagger}$ Professor, Department of Mathematics, AIAA Senior Member

${ }^{\ddagger}$ Professor, Department of Mathematical Sciences.

$\S$ Aerospace Engineer, Atmospheric Flight and Entry Systems Branch, AIAA Member 


$\begin{array}{ll}D_{A} & \text { Aeroshell diameter }(\mathrm{cm}) . \\ D_{T} & \text { Torus diameter }(\mathrm{cm}) . \\ D_{T o t} & \text { Total diameter }(\mathrm{cm}) . \\ D_{T S} & \text { Tension shell diameter }(\mathrm{cm}) . \\ P & \text { Differential pressure }(\mathrm{kPa}) . \\ p_{t} & \text { Torus pressure }(\mathrm{kPa}) . \\ q & \text { Dynamic pressure }(\mathrm{kPa}) . \\ p_{s} & \text { Static pressure }(\mathrm{kPa}) . \\ \alpha & \text { Angle of attack }(\mathrm{deg}) . \\ R_{N} & \text { Aeroshell nose radius }(\mathrm{cm}) . \\ M & \text { Mach number. } \\ \text { A-T } & \text { Anti-torque. } \\ \text { OTCP } & \text { Observed torus collapse pressure }(\mathrm{kPa}) . \\ \text { AARD } & \text { Absolute relative difference. } \\ \text { AARD } & \text { Average absolute relative difference. } \\ p_{c r} & \text { Critical torus pressure }(\mathrm{kPa}) . \\ \text { FSI } & \text { Fluid structure Interaction. }\end{array}$

\section{Introduction}

The Mars Science Laboratory (MSL) utilized the largest diameter aeroshell $(4.5 \mathrm{~m})$ and the largest diameter parachute ever flown on Mars to decelerate safely the $899 \mathrm{~kg}$ rover Curiosity to the surface of Mars. This system is near the limits of current parachute technology and future missions with larger landed mass may find it necessary to resort to a different decelerator technology. ${ }^{1,2}$ One alternative is the inflatable aerodynamic decelerator (IAD). The main advantage of IADs over parachutes is that they do not have the inflation instabilities that parachutes suffer at higher Mach numbers $(M>\sim 1.5)$. While inflatable aerodynamic decelerator (or ballute) technology dates back to the 1960 's ${ }^{3-6}$ in recent years due to the advantages offered by the IAD, this technology has garnered additional attention through NASA's Program to Advance Inflatable Decelerators for Atmospheric Entry (PAIDAE). ${ }^{7-9}$

An inflatable decelerator known as a tension-cone IAD (see Figure 1) has received particular attention. The key components of a tension cone IAD are a rigid blunt aeroshell, a tension shell designed to carry tensile loads, a pressurized torus, and in some versions, anti-torque (A-T) panels. In this paper, we will focus our attention on two tension-cone IAD physical models as presented by the third author and his collaborators. ${ }^{7,8,10}$ In this model, the tension shell and torus were constructed from urethane-coated kevlar. The A-T panels were introduced because, initially there 


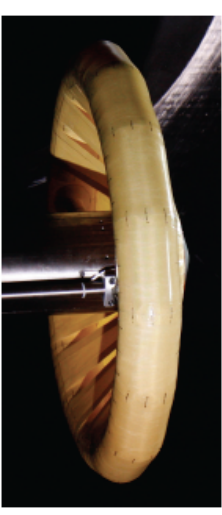

(a) With A-T panel

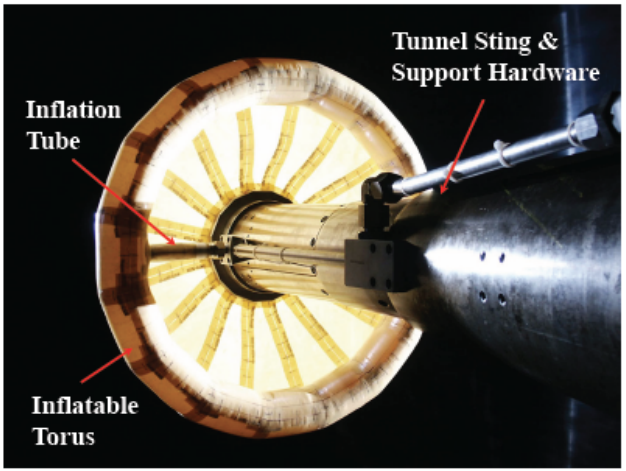

(d) Tension Cone IAD without A-T panels.

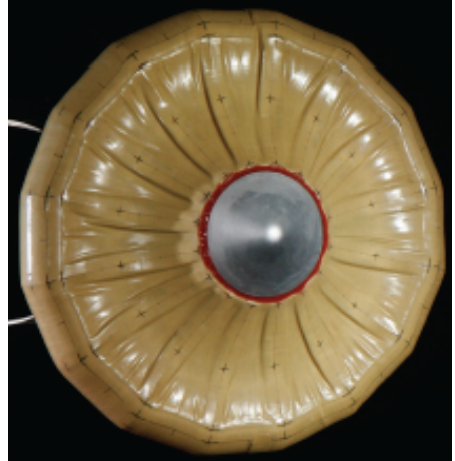

(c) Aerodynamically loaded IAD with A-T panels.

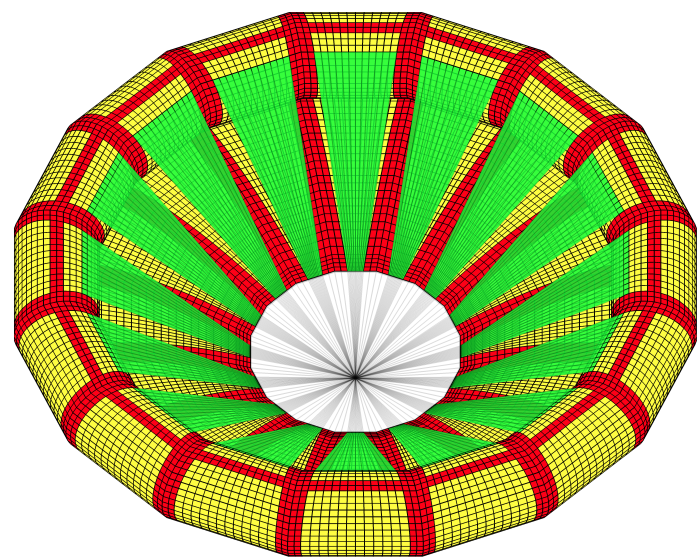

(e) Computer model of tension-cone IAD with A-T panels (aft view).

Figure 1. Tension-cone IAD models; (a) With A-T panel: (b) Without A-T panel; (c) Aerodynamically loaded with A-T panels (front view); (d) inflatable model with inflation tube, inflatable torus and support hardware; (e) computer model of an inflatable tension-cone IAD. Figures (a)-(d) correspond to $[10$, Figures 55(b), 55(a), 54, 62(a), respectively].

were concerns that radially compressive forces generated by the tension shell would cause the inflated torus to roll outward and forward. Figure 1(a) shows an IAD model with A-T panels and Figure 1(b) shows one without A-T panels. During wind tunnel testing, ${ }^{8,10}$ the models with and without A-T panels were found to behave very differently; we will discuss these differences in the following sections. Based on the published data and specifications, ${ }^{7,8}$ we were able to able to develop a mathematical model ${ }^{11,12}$ for the tension-cone IAD, including the membrane tension shell, torus, and A-T panels. See Figure 1(e) where membrane components are yellow, A-T panels are in green and seam reinforcements are shown in red.

One of the basic questions for aerodynamically loaded inflatable structures, in general, and 


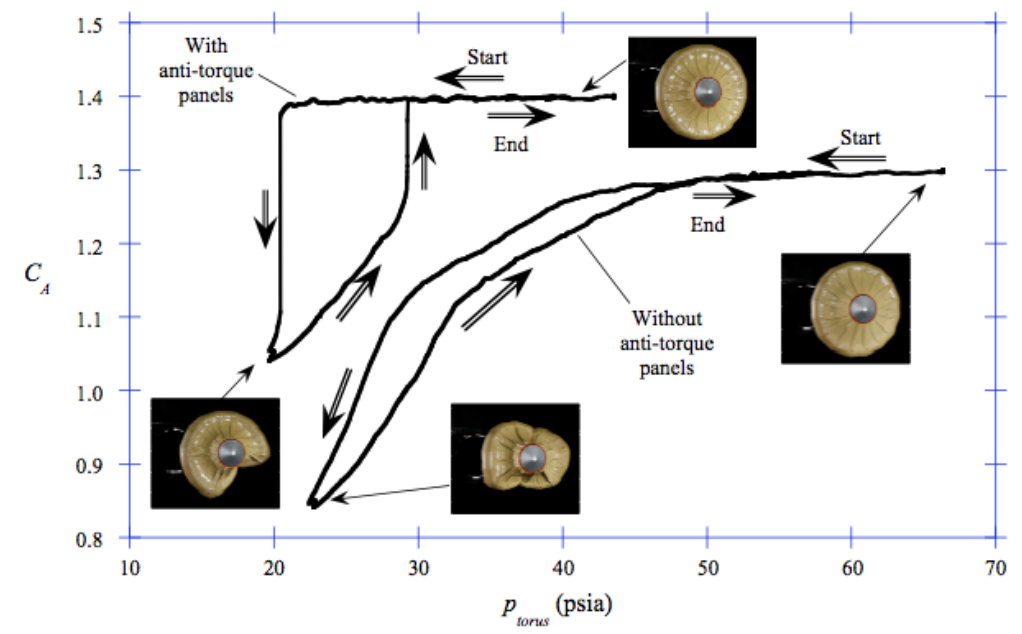

Figure 2. Pressure sweeps for GRC SWT inflatable models. Reproduced from [8, Fig. 14].

tension-cone IADs, in particular, is the following: what is the minimum differential pressure that is needed to maintain a certain desired aerodynamic shape? In the case of the IAD, the answer to this question will help the engineer meet design requirements for a lightweight deployable structure that packs into a small volume, is stiff enough to maintain a desired aerodynamic shape and enable the landing of larger payloads at higher altitudes. Roughly speaking, if the aerodynamic loading is not too extreme and the torus pressure is sufficiently large, the previously mentioned research ${ }^{7,8,10}$ have demonstrated through physical models and numerical computation that the tension-cone IAD shape is stable. However, if the aerodynamic pressure is maintained at a constant level and the torus pressure is reduced a sufficient amount, there is a pressure threshold where the torus will collapse. We call this particular pressure threshold the observed torus collapse pressure (OTCP). The instance of torus collapse has been observed in supersonic wind tunnel tests that involve pressure sweeps. A pressure sweep test has two parts. In the first part, the torus was inflated initially to a maximum pressure $p_{t, \max }$. While the tunnel operating conditions were maintained, the torus pressure was gradually reduced until the torus collapsed. In the second part of the pressure sweep test, the torus was re-inflated. The instance where the IAD recovered to the axisymmetric shape defined the observed torus re-inflation pressure. Researchers, including the third author, who were involved in the 2008 NASA Glenn Research Center Supersonic Wind Tunnel (GRC SWT) test estimated both the torus collapse threshold and the torus re-inflation pressure from the experimental 
data. In the pressure sweeps with the A-T panel model, there was a rapid change in the measured value of $C_{A}$ that could be correlated with the torus pressure which was recorded simultaneously. ${ }^{10}$ Hence, the torus collapse pressure was well-defined for the model with A-T panels. See Figure 2. However, since there was no sudden collapse of the test articles without A-T panels during the pressure sweeps and $C_{A}$ changed gradually, the instance of torus collapse is not well-defined. In fact, the definition of collapse pressure is somewhat arbitrary in the model without A-T panels.

It should be noted that some of the axial force on the model is reacted by the inflation tubes (see Figure 1(d)) and not measured by the wind tunnel balance. Thus, the value of $C_{A}$ reported here is not the same as would be obtained in free flight or in a static wind tunnel test without inflation tubes. However, since the quantity of interest in this paper is the value of the torus pressure at which the axial force exhibits a significant change, the absolute value of $C_{A}$ is not critical to the present discussion. Values of $C_{A}$ that are not affected by inflation tubes may be found by consulting other references. ${ }^{8,10}$ Nevertheless, at least the torus collapse pressure is well-defined in the model with A-T panels. The models with A-T panels (starting from a collapsed state) would change shape by a small amount, but remain in a collapsed state, as the torus pressure was increased. At the re-inflation pressure, they would pop up to the fully inflated shape, and the axial force would jump up. In the model without A-T panels, the torus re-inflation pressure was gradual and thus a threshold was not well-defined in these cases. The third author and Clark ${ }^{10}$ developed a heuristic method for estimating the observed torus collapse by correlating its occurrence with a change in the axial force coefficient $C_{A}$, a measured quantity (see, e.g., [8, Fig. 5]). Based on the test data and keeping in mind the above remarks, the $C_{A}$ threshold collapse was defined as 0.97 times the static value of $C_{A}$ that was recorded at full inflation pressure. For the second part of the same inflation test and after the torus collapsed, the torus was then re-inflated while wind tunnel conditions were maintained. This method of estimating OTCP worked well for the model with A-T panels (where there was a rapid change in $C_{A}$ ) and not so well for the model without A-T panels (where the change in $C_{A}$ was gradual).

There are subtleties in how the re-inflation pressure was determined, and the behavior of the models with and without anti-torque panels were very different. This can be seen in Figure 2 
(reproduced from [8, Fig. 14]). The values for OTCP presented in this paper include unpublished data from the GRC SWT tests carried out in April 2008. Plots for other pressure sweeps from the 2008 GRC SWT testing were very similar to Figure 2 (i.e., the results for the IAD model with A-T panels led to a well-defined torus collapse pressure, but for the model without A-T panels this was not the case). In general, the observed torus re-inflation pressure was significantly higher than the OTCP, but the OTCP and the inflation-to-collapse portion of the pressure sweeps was more pertinent to $p_{c r}$. The numerical modeling in ${ }^{10,13}$ performed well in predicting the behavior of the IAD without A-T panels, but not so well in the cases with A-T panels. We will discuss these comparisons in Sections III(C) and D.

\section{The Critical Pressure in Other Applications}

In previous articles ${ }^{14,15}$ the first two authors developed a methodology, called the Deployment Pathway Portrait (DPP), to predict the differential pressure at which an ascending super-pressure balloon is likely to deploy. A properly designed symmetric balloon in equilibrium in the fully deployed and fully pressurized state is stable. At float (maximum) altitude, the design volume is $V_{d}$ and the the differential pressure at the bottom of the balloon is $p_{d}>0$. At sea level, the volume of the lifting gas contained within the balloon envelope is compressed typically to less than $1 \%$ of $V_{d}$. The differential pressure at the bottom of the balloon is $p_{0}<0$. As the balloon rises, the volume of the lifting gas expands and its density decreases. Initially, the majority of the envelope hangs loosely beneath the gas bubble. The mass of the lifting gas is constant (although some gas may be vented during ascent). Eventually, the gas will expand a sufficient amount so that $p_{0}>0 ; p_{0}$ will continue to increase, until the balloon reaches its maximum altitude. The lowest value of $p_{0}$ at which a super-pressure balloon deploys is called the deployment pressure. The deployed state is one where the balloon is free of self contact (as determined by an observer who records a differential pressure when deployment is first observed). Typically, a super-pressure balloon deploys when $p_{0}<p_{d}$. The balloon continues to ascend and the pressure at the bottom of the balloon continues to increase until maximum altitude is achieved and $p_{0}=p_{d}$. It is only when $p_{0}=p_{d}$ that the super-pressure balloon assumes the fully developed pumpkin-shape. 
Using the second author's program Surface Evolver, ${ }^{16}$ we determined the strained equilibrium state of a single gore in a cyclically symmetric balloon shape and then carried out a stability analysis of the complete shape. For some $p_{0}=p_{c r}\left(0<p_{c r}<p_{d}\right)$, the stability of the symmetric state changed from unstable to stable. The threshold $p_{0}=p_{c r}$ where the symmetric state changed from stable to unstable is called the critical pressure (this is analogous to the critical torus pressure for the inflatable IAD). The critical pressure correlated well with the observed deployment pressure during tests and balloon flights. The family of cyclically symmetric ascent shapes $\mathcal{S}\left(p_{0}\right)$ parametrized by $p_{0}$ along with a count of the number of corresponding unstable eigen-modes is called the DPP. The DPP approach was validated for super-pressure balloons by comparing predicted deployment pressures with those that were recorded in ground-based inflation tests [14, Tables 3-5] and actual flight data [14, Tables 6-7].

In a recent article $[12$, Sec. III], the DPP approach was adapted to the problem of determining the critical torus pressure for conditions corresponding to the two pressure sweeps described in Figure 2. The DPP-based results were encouraging and the estimated critical torus pressure of $138 \mathrm{kPa}(19.9 \mathrm{psi}$ ) compared well with the observed torus collapse pressure (OTCP) of $120 \mathrm{kPa}$ (17.4 psi) for the tension-cone IAD model with A-T panels [12, Table 4]. While the absolute relative difference $(\mathrm{ARD}): \mathrm{AARD}=\left|p_{c r}-\mathrm{OTCP}\right| / p_{c r}$ of $13 \%$ for the $\mathrm{A}-\mathrm{T}$ panel case was good, when the anti-torque panels were removed, the predicted critical pressure of $236 \mathrm{kPa}(34.2 \mathrm{psi})$ was off by $24 \%$ when compared with a "conservative" OTCP that was estimated to lie in the interval $(310 \mathrm{kPa}, 345$ $\mathrm{kPa}$ ) (i.e., (45 psi, $50 \mathrm{psi})$ ). However, as we have discussed earlier, the OTCP is not well-defined in the case of the IAD model without A-T panels. Nevertheless, these preliminary findings provided the motivation to analyze additional data that was available from the NASA GRC SWT tests conducted in April 2008.

In this paper, we validate our mathematical approach for estimating the torus collapse pressure of an aerodynamically loaded tension-cone inflatable aerodynamic decelerator by comparing the predicted critical torus pressure with the observed torus collapse pressure for a total of fifteen pressure sweeps that were conducted in April 2008 at the NASA GRC SWT. The observed torus collapse pressure must be well-defined in order for these comparisons to be meaningful. We will vary 
the Mach number, dynamic pressure, static atmospheric pressure and torus differential pressure and carry out stability analyses of the symmetric equilibrium shapes. When the observed torus collapse pressure is well-defined (i.e., the IAD model with anti-torque panels), we found good agreement with the DPP predicted critical torus pressure. In particular, we found the relative difference between $p_{c r}$ and OTCP ranged between $1.1 \%$ and $15.7 \%$, with an average relative difference of $8.9 \%$. When anti-torque panels are not included in the model, the torus does not instantaneously collapse and it is difficult to define precisely the torus collapse pressure. Nevertheless, when using the value of the observed torus collapse estimated by the third author, we find the relative difference between $p_{c r}$ and OTCP ranged between $10.4 \%$ and $27.4 \%$, with an average relative difference of $18.1 \%$.

\section{Mathematical Model for a Tension-Cone IAD}

The tension cone inflatable aerodynamic decelerator (IAD) was proposed by Anderson et al. ${ }^{3}$ Additional details on the development of the IAD concept may be found in [13, Sec. 1.2.2]. The tension cone IAD is a shell of revolution consisting of a blunt aeroshell nose, a pressurized toroidal toroidal membrane and a a tension shell designed to carry tensile loads when acted upon by a known pressure field. See Figure 1(a)-(d) for photographs of IAD models and Figure 1(e) for a computer model of an IAD with A-T panels. Our IAD mathematical model is presented in Sections III.A-B (see also [12, Secs. III.A-B]).

\section{A. The GRC SWT IAD Model}

We will focus on the model relevant to the NASA GRC SWT IAD and use the design specifications in the article by Clark, et al. ${ }^{8}$ In the following, refer to Figure 3, where the generating curve for the GRC SWT IAD is presented. The legend in Figure 3 indicates four subregions: $\Omega_{T C}$ - tension cone and aeroshell nose; $\Omega_{T_{1}}$ - upstream side of torus; $\Omega_{T_{2}}$ - downstream side of torus; $\Omega_{A T P}$ anti-torque panel. Differential pressure $P(r, z)$ is a function of the radial distance $r$ and $z$. The dynamic pressure is treated as a static aerodynamic pressure distribution that is included in $P$. In 


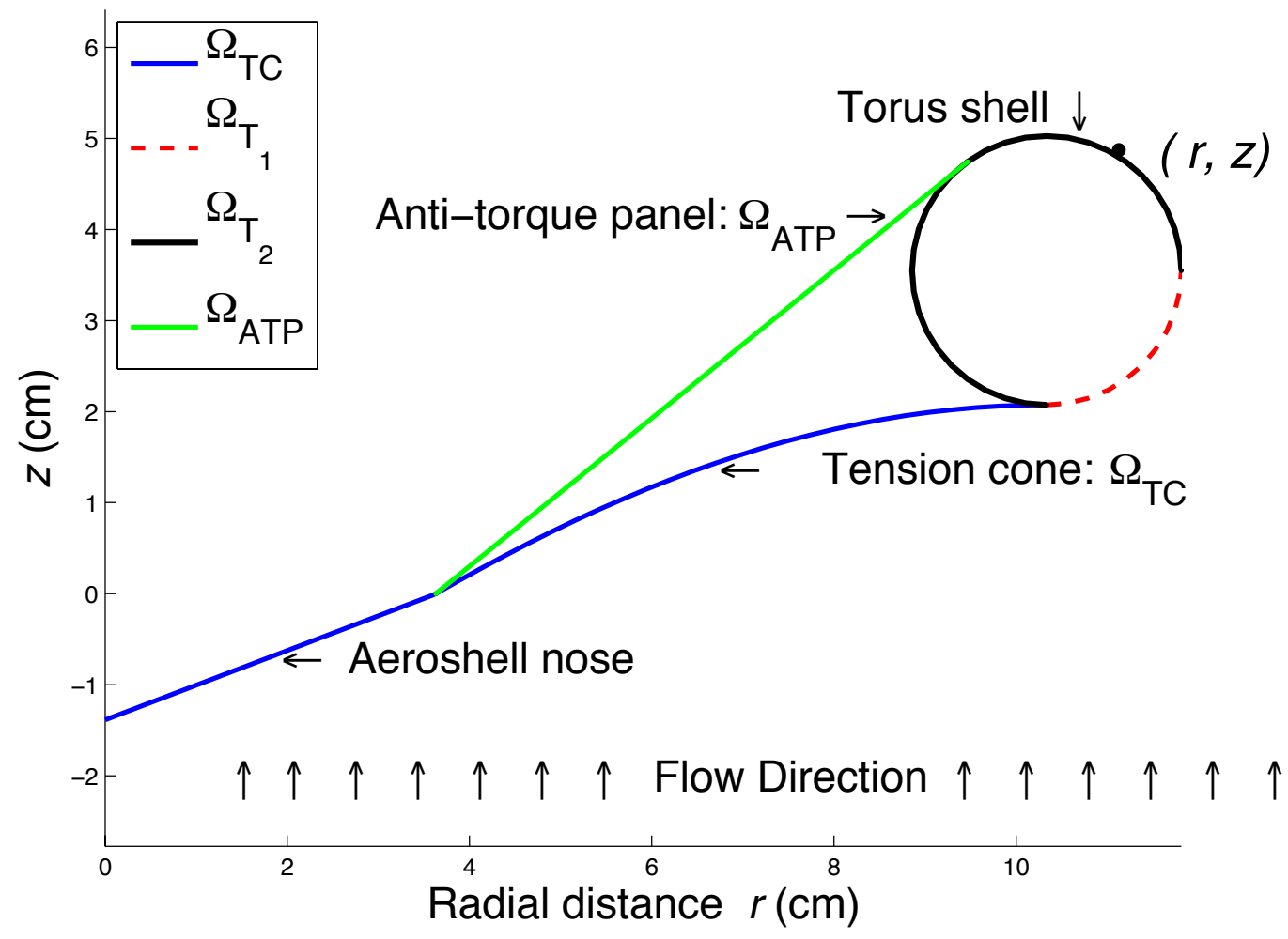

Figure 3. Generating curve for GRC SWT IAD shell of revolution.

the nominal case where we assume zero angle of attack (i.e., $\alpha=0), P$ is given by

$$
P(r, z)=\left\{\begin{array}{lll}
\left(c_{p, u}-c_{p, d}\right)(r) q & \text { on } \Omega_{T C} & \text { (Tension cone), } \\
c_{p, u}(r) q+p_{s}-p_{t} & \text { on } \Omega_{T_{1}} & \text { (Upstream torus) } \\
c_{p, d}(r) q+p_{s}-p_{t} & \text { on } \Omega_{T_{2}} & \text { (Downstream torus) } \\
0 & \text { on } \Omega_{A T P} & \text { (Anti-torque panels) }
\end{array}\right.
$$

where the regions $\Omega_{T C}, \Omega_{T_{1}}, \Omega_{T_{2}}$ and $\Omega_{A T P}$ are defined in Figure 3 and the differential pressure coefficients $c_{p, u}(r), c_{p, d}(r)$ are defined in Fig. 4. Fig. 4 is based on data from [8, III. Rigid Models Tests - LaRC UPWT] involving a $15.24 \mathrm{~cm}$ diameter physical model for Mach number $M=2.5$. $c_{p, u}(r)$ and $c_{p, d}(r)$ are the upstream and downstream coefficients, respectively. The data is rescaled so that it can be applied to the $60 \mathrm{~cm}$ diameter fabric model that is considered here. The aeroshell nose portion of $\Omega_{T C}$ is fixed. The values of $P$ depends on the angle of attack $\alpha$ and Eq. (1) will need to be adjusted if $\alpha \neq 0$. In fact, the data in Fig. 4 is for $\alpha=0.3 \mathrm{deg}$, but this is sufficiently close to zero for our purposes. We also note that when $\alpha \neq 0$, the flow is not axisymmetric. Mechanical properties for the membrane components of the IAD were assumed to be the same as those presented in ${ }^{7}$ and $[13$, Appendix A, Table A.2]. See Table 1 for a summary of parameter 


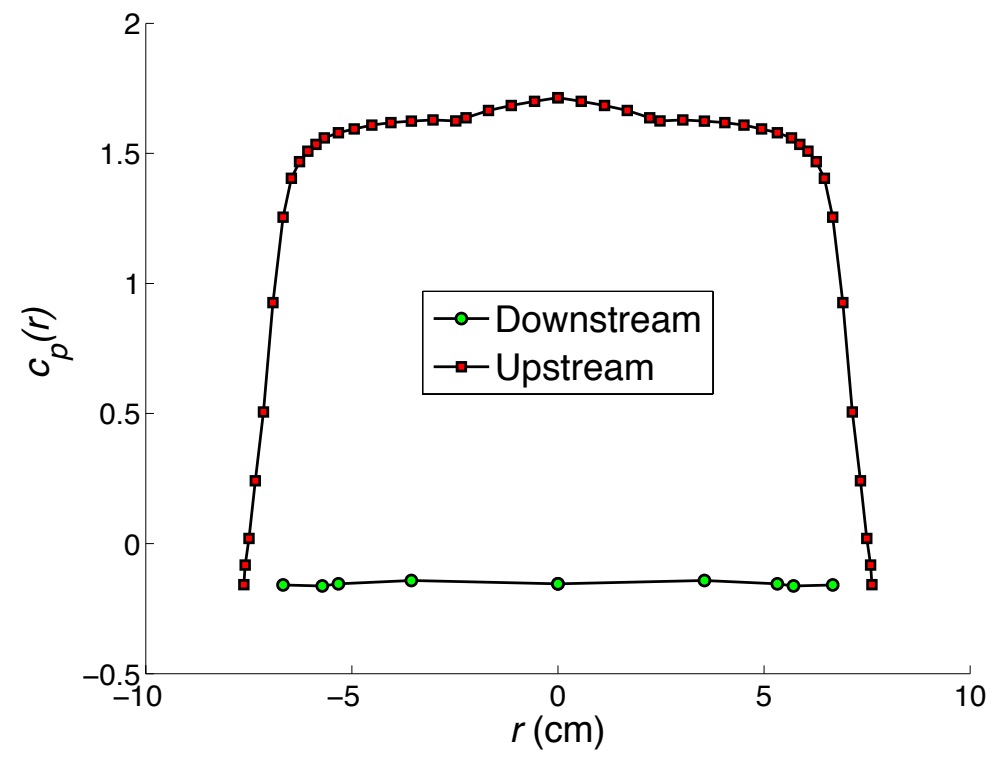

Figure 4. Pressure coefficient as a function of the radial coordinate $r$.

values. The surface pressures act on the IAD membrane. Torus pressures are absolute. We include anti-torque panels as depicted in [8, Fig. 12]. In case studies considered here, we add thickness along the gore component seams to bring our numerical model closer in line with the physical model for which experimental data is available. Note, the IAD model considered here is similar to, but not identical to, the IAD model in previous works. ${ }^{1,7,8,19}$ For example, inflation tubes are not considered in the model presented here. Nevertheless, the IAD model presented here is sufficiently close to the physical IAD model to evaluate our analytical methods.

Table 1. GRC SWT semi-rigid inflatable model. See [7, Table 1].

\begin{tabular}{ccc}
\hline Quantity & Variable & Value \\
\hline Young's modulus & $E$ & $2.67 \mathrm{GPa}$ \\
Poisson's ratio & $\nu$ & 0.3 \\
Thickness & $h$ & $334 \mu \mathrm{m}$ \\
Aeroshell diameter & $D_{A}$ & $18.44 \mathrm{~cm}$ \\
Torus diameter & $D_{T}$ & $7.5 \mathrm{~cm}$ \\
Total diameter & $D_{T o t}$ & $60.0 \mathrm{~cm}$ \\
Tension shell diameter & $D_{T S}$ & $52.5 \mathrm{~cm}$ \\
Angle of attack & $\alpha$ & $0 \mathrm{deg}$ \\
Aeroshell nose radius & $R_{N}$ & $4.74 \mathrm{~cm}$ \\
\hline
\end{tabular}

A true torus is generated by rotating a circle of radius $r$ about a line belonging to the plane of 


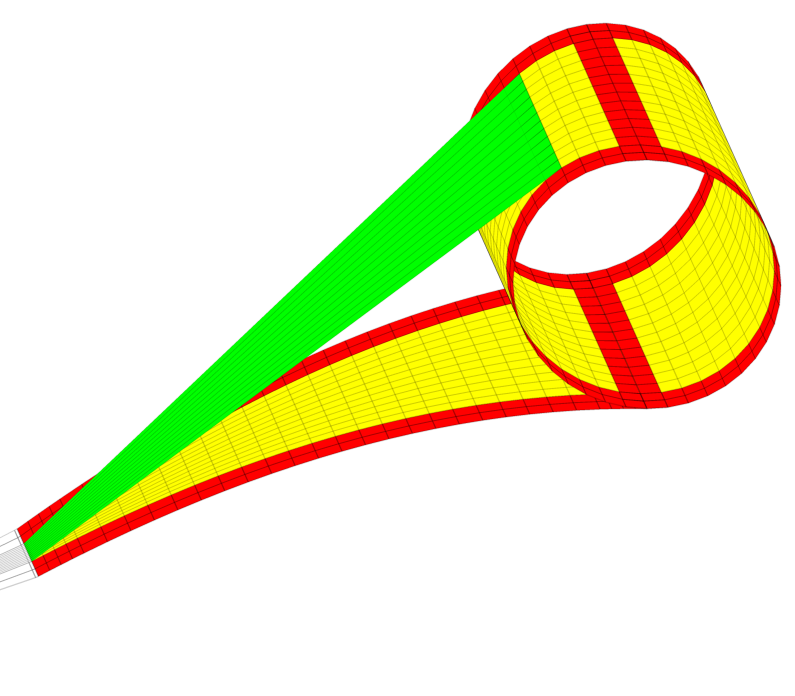

(a) $\mathcal{S}_{F}$ - fundamental section.

\section{Anti-Torque Panel}

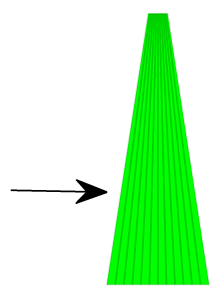

Torus

Tension Shell

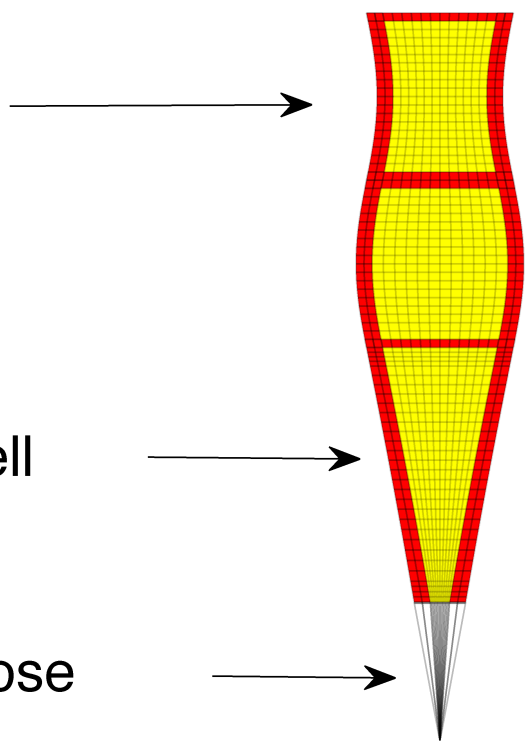

\section{Aeroshell Nose}

(b) $\mathcal{G}_{F}$ - reference configuration.

Figure 5. Reference configuration and fundamental section of torus IAD model. 
the circle and at a distance $a>r$ away from the center of the circle. Instead, we consider a straightsided torus IAD model generated in the following way. Assume the curve in Figure 3 lies in the plane $y=\tan (\pi / 16) x$ in three-dimensional Euclidean space $\mathbb{R}^{3}$. Project that curve orthogonally on to the plane $y=0$. The lines generated by the projection determine a developable surface. Reflect that developable surface in the plane $y=0$ and the result is a section of a straight-sided torus (i.e., a section of a cylinder) sandwiched between the planes $y= \pm \tan (\pi / 16) x$ (see Figure $5(\mathrm{a})$ ). The straight-sided torus IAD model can be unrolled into a plane as illustrated in Figure 5(b). We double the membrane thickness along certain edges (see the dark regions in Figure 5) to simulate reinforced seams (a seam width of 0.885 inches $(2.248 \mathrm{~cm}$ ) is used). The seam width of $4.45 \mathrm{~cm}$ in [12, Sec. III.A] is a typographical error (a seam width of $2.248 \mathrm{~cm}$ was used in the calculations and so the reported numerical results are unchanged). The seam width of the physical model is 1 inch. ${ }^{13}$ The bottom portion of the gore presented in Figure 5(b) corresponds to the reference configuration of the aeroshell nose, which is fixed. The complete IAD is shown in Figure 1(e).

\section{B. IAD Problem Formulation and Stability}

In this section, we outline the problem of determining the equilibrium shape of a strained pressurized membrane under aerodynamic loading. Details of the model can be found in other references. ${ }^{11,17,18}$ For a complete shape $\mathcal{S} \subset \mathbb{R}^{3}$, the reference configuration is $\Omega=\cup_{i=1}^{n_{g}} G_{i}$ where $G_{i}$ is isometric to the cutting pattern $G_{F}$ that we assume is known (see, Figure 5(b)). In the numerical model, the rectangular regions are subdivided into triangular facets. In this case, the complete deformed shape is $\mathcal{S}=\cup_{i=1}^{n_{g}} \mathcal{S}_{i}$ where $\mathcal{S}_{i}$ is a deformation of $G_{i}$. In the IAD application of interest in this paper, $n_{g}=16$. If we restrict our attention to cyclically symmetric shapes, then an equilibrium configuration of a fundamental gore is denoted by $\mathcal{S}_{F}$. We describe our model as it applies to a complete shape $\mathcal{S}$.

The total potential energy of $\mathcal{S}$ is denoted by $f$ where

$$
\begin{aligned}
f & =\mathcal{E}_{P}+S_{m}^{*}, \\
\mathcal{E}_{P} & =\int_{\mathcal{D}} P d V=\int_{\mathcal{S}} P z \mathbf{k} \cdot \mathbf{n} d \sigma, \\
S_{m}^{*} & =\int_{\Omega} W_{m}^{*} d A,
\end{aligned}
$$


$d A$ is area measure in the lay-flat reference configuration, $\mathcal{E}_{P}$ is the pressure potential and $S_{m}^{*}$ is the total membrane relaxed strain energy. We follow the convention that the torus pressure $p_{t}$ acts in the direction of the outward unit normal $\mathbf{n}$. $\mathcal{D}$ is composed of the torus interior plus the volume directly below the IAD surface. The pressure at any point on the IAD is given by $P(r, z)$ as defined in Eq. (1) and Figure 3; $d \sigma$ is surface area measure in the strained surface and $W_{m}^{*}$ is the relaxed membrane strain energy density. This approach has been applied successfully to the analysis of superpressure balloons. ${ }^{11,17,18}$

To determine a strained equilibrium shape, we solve the optimization problem:

$$
\min _{\mathcal{S} \in X} f(\mathcal{S})
$$

where $X$ denotes the class of feasible shapes. ${ }^{11,18}$ This approach is particularly well-suited for the analysis of compliant structures. Our mathematical model has been implemented into the second author's program Surface Evolver (see [16, www.susqu.edu/brakke/evolver]), which is used to carry out stability analysis.

$\mathcal{S}$ is triangulated and without loss of generality we will refer to the faceted surface as $\mathcal{S}$. The degrees of freedom (DOF) in a complete faceted shape $\mathcal{S}$ are the $x, y, z$-coordinates of the nodes of triangular facets that are free to move. Let $\boldsymbol{x}=\left(x_{1}, x_{2}, \ldots, x_{N}\right)$ be a list of the DOF. Let $f(\boldsymbol{x})$ be the total energy of a faceted balloon configuration $\mathcal{S}=\mathcal{S}(\boldsymbol{x}) . D f(\boldsymbol{x})=\left[\partial f / \partial x_{j}\right], j=1, \ldots, N$ is the gradient of $f$ evaluated at $\boldsymbol{x}$, where $\partial f / \partial x_{j}$ denotes partial differentiation with respect to $x_{j}$. The Hessian of $f$ evaluated at $\boldsymbol{x}$ is the $N \times N$ matrix, $H_{f}(\boldsymbol{x})=D^{2} f(\boldsymbol{x})=\left[\partial^{2} f / \partial x_{i} \partial x_{j}\right], i, j=1, \ldots, N$. Stability is defined in the usual way.

Definition III.1 Let $\mathcal{S}=\mathcal{S}(\boldsymbol{x})$ be a solution of (5). We say $\mathcal{S}$ is stable if all the eigenvalues of $H_{f}(\boldsymbol{x})$ are positive. $\mathcal{S}$ is unstable if at least one eigenvalue of $H_{f}(\boldsymbol{x})$ is negative. The stability of $\mathcal{S}$ is indeterminate if the lowest eigenvalue of $H_{f}(\boldsymbol{x})$ is zero.

While the number of degrees of freedom in the complete shape $\mathcal{S}$ is large, one does not evolve $\mathcal{S}$. We first determine $\mathcal{S}_{F}$ and then use symmetry to determine the DOF of $\mathcal{S}$ and assemble $H_{f}(\mathcal{S})$. An algebraic problem is solved to determine the sign of the smallest eigenvalue of $H_{f}$ and the stability of $\mathcal{S}$. If the symmetric state is unstable, then one can determine alternate equilibria. ${ }^{12}$ 
When the torus pressure $p_{t}$ is sufficiently large, the cyclically symmetric equilibrium shape is stable. This leads to the following definition.

Definition III.2 The critical torus pressure $p_{c r}$ is the lowest torus pressure $p_{t}$ for which the corresponding Hessian matrix $H_{f}(\mathcal{S})$ is positive definite (i.e., all the eigenvalues of $H_{f}(\mathcal{S})$ are positive).

\section{IAD Numerical Results}

In this section, we will calculate $p_{c r}$ for a number of different loading scenarios for a tension-cone IAD with and without anti-torque panels. To support this research, J. R. Cruz and I. G. Clark made available a comprehensive set of pressure sweep data and methodology used to analyze it. ${ }^{20}$ The observed torus collapse as determined by these methods is compared with the critical torus pressure $p_{c r}$. A total of fifteen pressure sweeps are considered here.

For the inflation tests involving the model with anti-torque panels, the torus collapse pressure was easy to discern. For each of the eight cases considered, the torus was inflated to a certain maximum value (a higher dynamic pressure loading corresponded to a higher (initial) maximum torus pressure). Table 2 contains key parameters (Mach number, dynamic pressure, static pressure, maximum torus pressure) for GRC SWT pressure sweeps for IADs with anti-torque panels. Table 2 presents the critical torus pressure and observed torus collapse pressure (OTCP). We include the "collapsed-to-inflated" (C-to-I) re-inflation pressure for completeness. Similar results for pressures sweeps for models without anti-torque panels are presented in Table 3.

Table 2 considers the IAD model with anti-torque panels. There are basically three parameter settings which are repeated two or three times. We include the Average Absolute Relative Difference (AARD, i.e., the average of the relevant values of Absolute Relative Differences

$$
\mathrm{AARD}=\left|\mathrm{OTCP}-p_{c r}\right| / p_{c r}
$$

for the Inflated-to-Collapse portion of the pressure sweep tests. Due to test variability, the measured quantities $\left(M, q, p_{s}\right)$ are slightly different in each test. The difference between between the results for the repeated tests is due to test variability and can be seen by examining Figure 6 where OTCP is plotted versus $p_{c r}$. With the exception of the columns corresponding to $p_{c r}$ and AARD, 
all data reported in Tables 2-3 is taken from summary tables of the pressure sweep data. ${ }^{20}$ We will refer to the various pressure sweeps by the run number that was assigned during the test.

- $\left(M, q, p_{s}\right)=(2.42,2.5 \mathrm{kPa}, 0.6 \mathrm{kPa})$; Repeat two times; Run Numbers: 26, 137; AARD: $6.4 \%$.

- $\left(M, q, p_{s}\right)=(2.44,4.5 \mathrm{kPa}, 1.0 \mathrm{kPa})$; Repeat three times; Run Numbers: 28, 78, 136; AARD: $4.9 \%$.

- $\left(q, p_{s}\right)=(2.46,7.2 \mathrm{kPa}, 1.7 \mathrm{kPa})$; Repeat three times; Run Numbers: 30, 134, 145; AARD: $14.5 \%$.

Table 2. SWT pressure sweeps - model with anti-torque panels. Units of pressure: kPa.

\begin{tabular}{ccccccccc}
\hline Run & $M$ & $q$ & $p_{s}$ & $p_{t, \max }$ & $p_{c r}$ & OTCP & AARD & C-to-I \\
\hline 26 & 2.424 & 2.513 & 0.611 & 179.6 & 43.64 & 38.5 & $11.7 \%$ & 65.39 \\
137 & 2.428 & 2.515 & 0.610 & 164.1 & 43.67 & 43.20 & $1.1 \%$ & 69.45 \\
136 & 2.451 & 4.518 & 1.074 & 245.0 & 78.17 & 84.38 & $7.9 \%$ & 133.3 \\
28 & 2.444 & 4.538 & 1.053 & 246.1 & 78.51 & 81.41 & $3.7 \%$ & 120.1 \\
78 & 2.439 & 4.579 & 1.099 & 426.9 & 79.24 & 81.86 & $3.3 \%$ & 125.1 \\
30 & 2.458 & 7.207 & 1.704 & 289.6 & 123.4 & 140.8 & $14.1 \%$ & 201.6 \\
134 & 2.467 & 7.223 & 1.695 & 261.7 & 123.7 & 143.1 & $15.7 \%$ & 220.4 \\
145 & 2.468 & 7.229 & 1.700 & 236.6 & 123.8 & 141.0 & $13.9 \%$ & 222.0 \\
\hline
\end{tabular}

Table 3 considers the IAD model with no anti-torque panels. Three parameter settings are repeated two or three times. In the case without anti-torque panels, the instance of torus collapse is not well defined and the torus collapses over an interval of pressure. See "Without anti-torque panels" in Figure 2. Nevertheless, to estimate an instance of torus collapse, we can utilize the axial force coefficient approach. ${ }^{20}$ These estimates for observed torus collapse are presented in Table 3.

- $\left(M, q, p_{s}\right)=(2.43,2.5 \mathrm{kPa}, 0.6 \mathrm{kPa})$; Repeat two times; Run Numbers: 37, 153; AARD: $23.5 \%$

- $\left(M, q, p_{s}\right)=(2.45,4.5 \mathrm{kPa}, 1.1 \mathrm{kPa})$; Repeat three times; Run Numbers: 39, 51, 154; AARD: $17.8 \%$.

- $\left(M, q, p_{s}\right)=(2.47,7.2 \mathrm{kPa}, 1.7 \mathrm{kPa})$; Repeat three times; Run Numbers: 41, 152; AARD: $13.1 \%$.

Figure 6 presents a plot of observed torus collapse pressure versus critical torus pressure. Data for IADs with and without A-T panels are included. The line OTCP $=p_{c r}$ is also presented. It is clear from the above observations and those noted in [12, Sec. III.C.3], it is difficult identify a 
Table 3. SWT pressure sweeps - model with no anti-torque panels. Units of pressure: kPa.

\begin{tabular}{ccccccccc}
\hline Run & $M$ & $q$ & $p_{s}$ & $p_{t, \max }$ & $p_{c r}$ & OTCP & AARD & C-to-I \\
\hline 37 & 2.427 & 2.507 & 0.608 & 228.8 & 71.59 & 85.61 & $19.6 \%$ & 101.4 \\
153 & 2.428 & 2.509 & 0.608 & 402.0 & 71.60 & 91.22 & $27.4 \%$ & 108.9 \\
39 & 2.453 & 4.574 & 1.086 & 293.8 & 145.6 & 168.4 & $15.6 \%$ & 190.5 \\
154 & 2.453 & 4.574 & 1.086 & 266.4 & 145.6 & 177.1 & $21.6 \%$ & 198.8 \\
51 & 2.453 & 4.579 & 1.091 & 426.9 & 145.8 & 169.6 & $16.3 \%$ & 191.1 \\
41 & 2.470 & 7.205 & 1.688 & 457.8 & 235.4 & 272.8 & $15.9 \%$ & 304.1 \\
152 & 2.468 & 7.233 & 1.696 & 399.5 & 236.6 & 261.2 & $10.4 \%$ & 316.2 \\
\hline
\end{tabular}

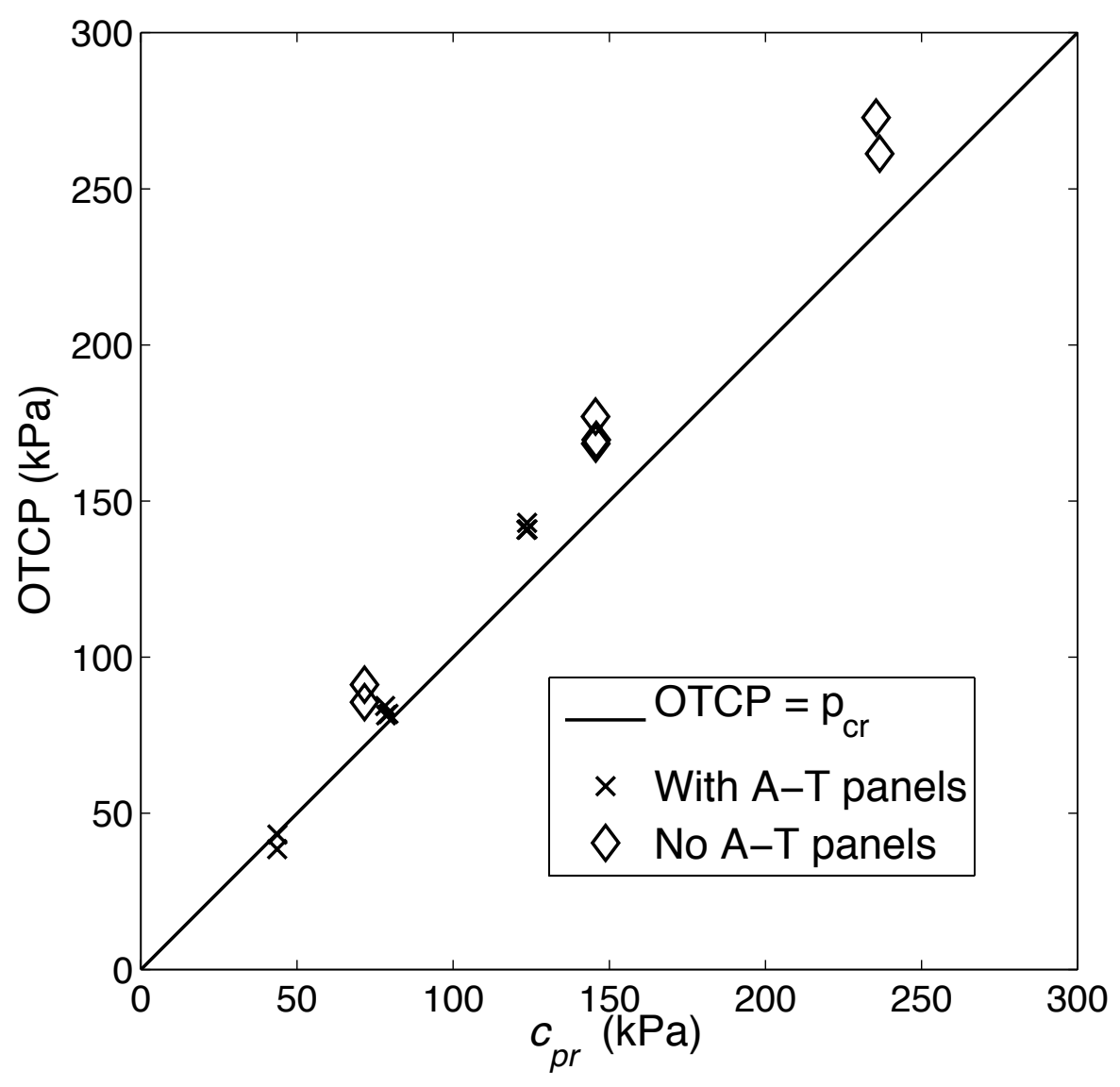

Figure 6. OTCP versus $p_{c r}$.

unique criteria that does not have some level of arbitrariness to define the torus collapse pressure for the IAD model with no anti-torque panels. On the other hand, the instance of torus collapse for the IAD model with anti-torque panels is more clearly defined due to the sharp change in the axial force coefficient.

In [12, Fig. 11], we estimated that the torus collapse pressure to be $138 \mathrm{kPa}$ for Case 30 and 
between $310 \mathrm{kPa}(45 \mathrm{psi})$ and $345 \mathrm{kPa}$ (50 psi) for Case 41 by extrapolating from Figure 2. This method of estimating the observed torus collapse pressure is prone to introduce errors. To address this issue, a heuristic method $^{8,10}$ for estimating the observed torus collapse by correlating its occurrence with a change in the axial force coefficient $C_{A}$ was proposed (see, e.g., [8, Fig. 5]). Based on the test data, the $C_{A}$ threshold collapse was defined as 0.97 times the static value of $C_{A}$ that was recorded at full inflation pressure. ${ }^{10}$ The collapse pressure that was associated with OTCP and C-to-I are distinguished, but we compute a single critical torus pressure $p_{c r}$. Table 3 contains similar information for the pressure sweeps without anti-torque panels. The "observed" torus collapse pressures reported in Table 2-3 were determined from the axial force coefficient measurements. $^{8,10}$

Using a Fluid Structure Interaction (FSI) model, Tanner estimated the torus collapse pressure to be $287 \mathrm{kPa}$ (41.6 psi) [13, Sec. 4.7.1] for Case 41; using a FSI model to correct the axial force coefficient data, the FSI corrected observed torus collapse pressure was $290 \mathrm{kPa}$ (42.1 psi), leading to a relative error of $1.1 \%$. Tanner notes the FSI analysis of the tension-cone IAD with antitorque panels was hindered by the collapse of the space between the torus and the tension shell, preventing more than one FSI iteration [13, p. 193]. When anti-torque panels are included in the model, Tanner estimated the torus collapse pressure to be $261 \mathrm{kPa}(32.8 \mathrm{psi})$ [13, Sec. 4.7.2] for Case 30, significantly higher than the observed torus collapse pressure of $141 \mathrm{kPa}(20.5 \mathrm{psi})$. It should be pointed out that without anti-torque panels, Tanner's finite element model results and the observed test article are in very good agreement. See [13, Fig. 4.48] where there is a striking qualitative similarity between the FEM solution and test article without anti-torque panels. This was not the case when anti-torque panels are included in the model.

The DPP method only requires the calculation of a fundamental piece (i.e., one-sixteenth) of the complete shape as the torus pressure is gradually reduced. However, the stability of the complete symmetric shape is assessed at each step. While an axisymmetric state can always be found, at the critical torus pressure threshold its stability changes from stable to unstable and as the results of the previous section demonstrates, this change of stability is an indication of torus collapse. Unlike the FEM or FSI approaches, we need not compute a complete non-symmetric equilibrium state of 
the IAD as we approach the instability threshold. This is the reason the DPP method works well for predicting the torus collapse for the IAD model with anti-torque panels.

\section{Discussion of Numerical Results}

In this paper, we validate our mathematical approach for estimating the torus collapse pressure of a tension cone inflatable aerodynamic decelerator by comparing the computed critical torus pressure with the observed torus collapse pressure from GRC SWT testing that was carried out in 2008. We varied Mach number, dynamic pressure, static atmospheric pressure and torus differential pressure and carried out stability analyses of the symmetric equilibrium shapes. In general, we find good agreement between the DPP predicted $p_{c r}$ and the observed torus collapse pressure for the model with anti-torque panels. The average relative difference between predicted and observed torus collapse is about $8.9 \%$. When anti-torque panels are not included in the model, the torus does not instantaneously collapse and it is difficult to define precisely the torus collapse pressure. In this case, the average relative difference is about $18.1 \%$. Because the torus collapse pressures presented here are based on the axial force measurements from the tests, ${ }^{20}$ the relative differences are, in general, more reliable than those reported in [12, Table 4] which were extrapolated from the presentation in Figure 2. The results presented in this paper for tension-cone IADs with anti-torque panels and the successful application of the DPP method to the deployment of super-pressure balloons suggest the method may be applicable to other aerodynamic decelerators such as the Inflatable Reentry Vehicle Experiment (IRVE) ${ }^{19}$ or other deployable structures such as the ExaVolt Antenna (EVA): a large-aperture balloon-embedded antenna for ultra-high energy particle detection. ${ }^{21}$

\section{Conclusion}

The Deployment Pathway Portrait approach was applied to a tension cone inflatable aerodynamic decelerator under a variety of loading conditions. We conjectured that the torus collapse pressure of an inflatable aerodynamic decelerator coincides with the the lowest pressure for which the symmetric state is stable. The difference between predicted and observed torus collapse thresholds for an inflatable aerodynamic decelerator with anti-torque panels differed by about 8.9\%. The observed torus collapse threshold is is not as well-defined for the model without anti-torque panels 
and the difference between observed and predicted is about $18 \%$. These results are encouraging and suggest they may be applicable to other deployable structures such as Inflatable Reentry Vehicle Experiment (IRVE) and the ExaVolt Antenna (EVA).

\section{References}

${ }^{1}$ Clark, I. G., Hutchings, A. L., Tanner, C. L., and Braun, R.D., Supersonic Inflatable Aerodynamic Decelerators for Use on Future Robotic Missions to Mars, Journal of Spacecraft and Rockets, Vol. 46, No. 2, 2009, 340-352.

${ }^{2}$ Cruz, J. R., and Lingard, J. S., Aerodynamic Decelerators for Planetary Exploration: Past, Present, and Future, AIAA Paper 2006-6792, Aug. 2006.

${ }^{3}$ Anderson, M. S., Robinson, J. C., Bush, H. G., and Fralich, R. W., A Tension Shell Structure for Application to Entry Vehicles, NASA TN D-2675, 1965.

${ }^{4}$ Anon., PEPP Ballute Design and Development Final Report, NASA CR-66585, 1967.

${ }^{5}$ Barton, R. R., Development of Attached Inflatable Decelerators for Supersonic Application, NASA CR-66613, 1968.

${ }^{6}$ Mikulas, M. M., Jr., and Bohon, H. L., Development Status of Attached Inflatable Decelerators, Journal of Spacecraft and Rockets, 6, No. 6, June 1969, pp. 654-660.

${ }^{7}$ Tanner, C. L., Cruz, J. R., and Braun, R., Structural Verification and Modeling of a Tension Cone Inflatable Aerodynamic Decelerator, AIAA 2010-2830, 51st AIAA/ASME/ASCE/AHS/ASC Structures, Structural Dynamics, and Materials Conference, 12-15 April, 2010, Orlando, Florida.

${ }^{8}$ Clark, I. G., Cruz, J. R., Hughes, Ware, J. S. Madlanghayan, A. Braun, R. D., Aerodynamic and Aeroelastic Characteristics of a Tension Cone Inflatable Aerodynamic Decelerator, AIAA 2009-2967, 20th AIAA Aerodynamic Decelerator Systems Technology Conference, 3-7 May 2009, Seattle, WA.

${ }^{9}$ Clark, Ian G. and Braun, Robert D. and Ruffin, Stephen M., Validation of Computational Analyses for Supersonic Tension-Cone Static Aerodynamic Performance, Journal of Spacecraft and Rockets, 49 No. 3 (2012) 483-495.

${ }^{10}$ Clark, I. G., Aerodynamic design, analysis and validation of a supersonic inflatable decelerator, Doctoral Dissertation, Georgia Institute of Technology, August 2009.

${ }^{11}$ Barg, M., Lee, J., and Baginski, F., Modeling the Equilibrium Configuration of a Piecewise-Orthotropic Pneumatic Envelope with Applications to Pumpkin-Shaped Balloons, SIAM J. Appl Math., 71 (1) 2011, 20-40.

${ }^{12}$ Baginski, F. \& Brakke, K., Deployment of Pneumatic Envelopes Including Ascending Balloons and Inflatable Aerodynamic Decelerators, Journal of Spacecraft and Rockets, 49 No. 2 (2012) 413-421.

${ }^{13}$ Tanner, C. L., Aeroelastic analysis and testing of supersonic inflatable aerodynamic decelerators, Doctoral Dissertation, Georgia Institute of Technology, May 2012.

${ }^{14}$ Baginski, F. \& Brakke, K., Estimating the deployment pressure in pumpkin balloons, Journal of Aircraft, 48 No. 1 (2011) 235-247.

${ }^{15}$ F. Baginski, K. Brakke, \& R. Farley, Predicting the deployment pressure of an ascending pumpkin balloon, AIAA 2011-6831, 11th AIAA ATIO/NAVY 2011 Conference, 20-22 September 2011, Virginia Beach, VA.

${ }^{16}$ Brakke, K., The Surface Evolver, Experiment. Math. 1(2), 141-165, 1992.

${ }^{17}$ Baginski, F., Brakke, K. \& Schur, W., Unstable cyclically symmetric and stable asymmetric pumpkin balloon configurations, Journal of Aircraft, 44(3), 764-772, 2007.

${ }^{18}$ Baginski, F., Barg, M, Collier, W., Existence Theorems for Tendon Reinforced Thin Wrinkled Membranes Subjected to a Hydrostatic Pressure, Mathematics and the Mechanics of Solids, 13(6), 532-570, 2008.

${ }^{19}$ Lindell, M., Hughes, S., Dixon, M., and Wiley, C., Structural analysis and testing of the inflatable re-entry vehicle experiment (IRVE), AIAA 2006-16999, 47th AIAA/ASME/ASCE/AHS/ASC Structures, Structural Dynamics, and Materials Conference, 1-4 May 2006, Newport, RI.

${ }^{20}$ Personal communication. J. R. Cruz (NASA LaRC) and I. G. Clark (JPL) made available a comprehensive set of pressure sweep data and the methodology used to analyze it.

${ }^{21}$ Gorham, P. W., Baginski, F., Allison, P., Liewer, K. M., Miki, C., Hill, B. and Varner, G. S., The ExaVolt Antenna: A Large-Aperture, Balloon-embedded Antenna for Ultra-high Energy Particle Detection, Astroparticle Physics, 35 No. 5 (2011) 242-256. 